\title{
Abundância e biomassa de lianas em um fragmento de floresta Atlântica
}

\author{
Paula Guimarães Carvalho ${ }^{1}$, Juliano van Mellis ${ }^{2}$, Bruno Marques Ascenção ${ }^{3}$, Fernando Mancini Cestari ${ }^{3}$, \\ Luciana Ferreira Alves ${ }^{3,4}$ e Maria Tereza Grombone-Guaratini ${ }^{3,5}$
}

Recebido: 3.03.2011; aceito: 14.07.2011

\begin{abstract}
Liana abundance and biomass in an Atlantic forest fragment). Lianas represent an important component of the structure and diversity of tropical forests. Normally, in disturbed forest a higher density and biomass of lianas is found compared with undisturbed forests. The aim of this study was to evaluate if the abundance and biomass of lianas differ between bamboo-dominated and non-dominated areas in Parque Estadual das Fontes do Ipiranga. Sample plots of 0.7 ha were established in both areas, and a standard census protocol for woody lianas $\geq 1 \mathrm{~cm}$ were used. We found 277 lianas (395 ind. ha ${ }^{-1}$ ) in the bamboo-dominated area and 754 lianas $\left(1,077\right.$ ind. ha $\left.^{-1}\right)$ in the non-dominated area. The biomass, abundance and the proportion of infested trees were significantly different between areas. Lianas $\geq 5 \mathrm{~cm}$ diameter accounted for $75 \%$ of total biomass in the bamboo-dominated area and $21,4 \%$ in the non-dominated area. In bamboo-dominated area lianas may be suppressed by absence of supports and by competition with Aulonemia aristulata a native scandent bamboo.
\end{abstract}

Key words: Aulonemia aristulata, bamboo, forest fragment, lianas biomass

RESUMO - (Abundância e biomassa de lianas em um fragmento de floresta Atlântica). Lianas representam um importante componente da estrutura e diversidade das florestas tropicais. Normalmente em florestas perturbadas são encontradas uma alta densidade de lianas em comparação com florestas não perturbadas. O objetivo deste estudo foi avaliar se a abundância e a biomassa de lianas difere entre áreas dominadas e não-dominadas por uma espécie de bambu nativo no Parque Estadual das Fontes do Ipiranga. Uma parcela de 0,7 ha foi estabelecida em cada área e um censo com protocolo padrão para lianas lenhosas $\geq 1 \mathrm{~cm}$ foi usado. Nós encontramos 277 lianas ( 395 ind. ha ${ }^{-1}$ ) na área dominada por bambu e 754 lianas (1.077 ind. ha $\left.{ }^{-1}\right)$ na área não dominada. A biomassa e a proporção de árvores infestadas foram diferentes entre áreas. Lianas $\geq 5 \mathrm{~cm}$ de diâmetro foram responsáveis por $75 \%$ da biomassa total na área dominada por bambu e por $21,4 \%$ na área não dominada por bambu. Na área dominada por bambu as lianas podem ter sido suprimidas pela ausência de suporte e pela competição com Aulonemia aristulata um bambu nativo escandente.

Palavras-chave: Aulonemia aristulata, bambu, biomassa de lianas, fragmento florestal

\section{Introdução}

Lianas são um grupo polifilético de plantas lenhosas que possuem estratégias especializadas para ascenderem à copa da floresta usando a arquitetura de outras plantas como apoio mecânico (Schnitzer \& Bongers 2002). Estas plantas representam parte importante da estrutura e diversidade total das florestas tropicais e são componentes naturais deste tipo de comunidade (Engel et al. 1998). Embora este grupo de plantas seja abundante e diverso em florestas em todo o mundo, é nos trópicos onde se encontra maior abundância, riqueza e variedade de formas e tamanhos (Putz 1984, Schnitzer \& Bongers 2002). Em função disso, é nesta região que a maioria dos estudos com lianas tem sido conduzida, buscando elucidar seu papel na regeneração e manutenção da diversidade de espécies e nos processos ecológicos (Schnitzer et al. 2000, Granados \& Körner 2002, Wright et al. 2004).

1. Curso de Pós-Graduação em Biodiversidade Vegetal e Meio Ambiente, Instituto de Botânica, Caixa Postal 68041, 04045-972 São Paulo, SP, Brasil

2. Curso de Pós-Graduação em Biologia Vegetal, Universidade Estadual de Campinas, Caixa Postal 6109, 13083-970 Campinas, SP, Brasil

3. Instituto de Botânica, Núcleo de Pesquisa em Ecologia, Caixa Postal 68041, 04045-972 São Paulo, SP, Brasil

4. University of Colorado, INSTAAR, Campus Box 450, 80309 Boulder, CO, USA

5. Autor para correspondência: mgromboneguaratini@gmail.com 
Em grandes escalas espaciais, a abundância de lianas varia em função de fatores abióticos como a pluviosidade total, a sazonalidade da precipitação, a drenagem e a topografia do solo (Schnitzer \& Bongers 2002, DeWalt et al. 2010 ). Em escala local, a dominância varia em função da disponibilidade de suporte, da escala de distúrbio e da idade da floresta (Putz 1984, Senbeta et al. 2005, Madeira et al. 2009). De maneira geral, florestas secundárias, quando comparadas a florestas maduras, apresentam alterações estruturais e microclimáticas que favorecem o aumento na densidade e biomassa de lianas (Kapos et al. 1997, Laurance 1997, Tabarelli \& Mantovani 1999, Oosterhoorn \& Kappelle 2000, Schnitzer \& Bonger 2002). A abertura de clareiras e a presença de bordas favorecem a colonização por lianas uma vez que estas são mais eficientes na captura de recursos e no crescimento, o que lhes confere uma vantagem competitiva sobre as árvores nestes ambientes (Zhu \& Cao 2010) podendo, então, suprimir o crescimento de plântulas de espécies arbóreas e alterar a dinâmica florestal (Gentry \& Dodson 1987). Estimativas realizadas em florestas tropicais mostram que a biomassa de lianas varia de $1 \%$ a $13,7 \%$, sendo os maiores valores encontrados em florestas secundárias jovens (Sarmiento et al. 2005).

Potencialmente, lianas têm efeito negativo sobre as espécies arbóreas suprimindo o crescimento em diâmetro e aumentando o risco de morte devido ao peso excessivo para as árvores que as suportam (Schnitzer et al. 2000, Schnitzer \& Bongers 2002, Phillips et al. 2002, 2005, Malizia \& Grau 2006, van der Heijden et al. 2008, Ingwell et al. 2010). Entretanto, devido à alta razão entre folhas e caule, lianas são importantes para a biomassa foliar das florestas tropicais (Gentry 1983).

Apesar da importância das lianas na estrutura e composição das florestas tropicais, estudos envolvendo lianas têm recebido pouca atenção quando comparado aos com arbóreas (Letcher \& Chazdon 2009). Além disso, os modelos ecológicos que as incluem não são categóricos (Phillips et al. 2005) e os diferentes critérios usados nos levantamentos demográficos podem afetar as estimativas de abundância, área basal e biomassa (Schnitzer et al. 2006).

O Parque Estadual das Fontes do Ipiranga (PEFI), localizado no Município de São Paulo, é um dos poucos fragmentos remanescentes de Floresta Ombrófila Densa no domínio de Mata Atlântica (Veloso et al.
1991). Atualmente, o PEFI é a terceira maior reserva de vegetação nativa do Município de São Paulo (Barros et al. 2002), e está inserido na Reserva da Biosfera do Cinturão Verde da Cidade de São Paulo, considerada pela UNESCO como área de relevância ecológica mundial (Victor et al. 2004). Perturbações ocorridas no passado como extração de árvores, derrubada parcial de trechos da floresta e poluição, resultaram no aumento do número de clareiras e na descontinuidade do dossel (Pivello \& Peccinini 2002). Com isso, o PEFI apresenta áreas florestais secundárias em processo de regeneração, formando um mosaico de fases sucessionais distintas, onde áreas mais preservadas ocorrem adjacentes a áreas intensamente perturbadas e ocupadas por espécies dos estádios iniciais de sucessão como, por exemplo, Alchornea sidifolia Muell. Arg. e Cecropia glazioui Snethl (Pivello \& Peccinini 2002) e por várias espécies de bambus nativos.

Embora o PEFI tenha um histórico de perturbações, poucos estudos avaliaram o efeito desses distúrbios na estrutura (Pivello \& Peccinini 2002) ou na dinâmica da área (Gomes et al. 2002), e nenhum deles avaliou esse efeito na comunidade de lianas. Para que se possa estimar o papel das lianas sobre a regeneração florestal é preciso que inicialmente se conheça a localização e a intensidade de infestação de lianas sobre as árvores (Ladwig \& Miners 2010).

Bambus apresentam uma grande amplitude ecológica em resposta às perturbações do dossel (González et al. 2002). Assim como as lianas, bambus são bons competidores, podendo causar efeito negativo sobre a estrutura e dinâmica florestal (Griscon \& Ashton 2003, Campanello et al. 2005). A expectativa deste estudo é a de que a área dominada por bambus apresente menor densidade e biomassa de lianas em função da baixa disponibilidade de árvores para suporte e da competição com Aulonemia aristulata, um bambu nativo escandente. Nesse contexto, o objetivo deste trabalho foi avaliar se a abundância e a biomassa lenhosa de lianas diferem entre áreas com diferentes graus de perturbação do dossel e dominância por uma espécie nativa de bambu de hábito escandente, Aulonemia aristulata (Döll) Macclure. Especificamente foi avaliado: (1) a correlação entre a biomassa e densidade de árvores e a biomassa e a densidade de lianas e (2) a correlação entre o grau de abertura do dossel e a biomassa de lianas. 


\section{Material e métodos}

O estudo foi realizado no Parque Estadual das Fontes do Ipiranga (PEFI), um fragmento isolado de Floresta Atlântica (ca. 530 ha) localizado na região sudeste do Município de São Paulo, SP (233' 40”'S, $\left.46^{\circ} 36^{\prime} 38^{\prime \prime} \mathrm{W}\right)$ com cotas altimétricas variando entre 770 e 825 m (Nastri et al. 1992) e solo do tipo Latossolo Vermelho-Amarelo Distrófico (Embrapa 1999). O clima da região enquadra-se na categoria $\mathrm{Cwb}$ segundo a classificação de Köppen (1948), ou seja, temperado com verão chuvoso e inverno seco. As temperaturas médias mensais variam entre $15,7{ }^{\circ} \mathrm{C}$ (julho) e $22,4{ }^{\circ} \mathrm{C}$ (fevereiro). A precipitação média anual é de $1.540 \mathrm{~mm}$ com menos de $100 \mathrm{~mm}$ ao mês de precipitação de abril a setembro.

Cerca de 350 hectares do PEFI são cobertos por Floresta Tropical Baixo Montana. (sensu Oliveira Filho $\&$ Fontes 2000). O parque tem cerca de 380 espécies de árvores, sendo Myrtaceae, Fabaceae, Lauraceae e Melastomataceae as famílias mais abundantes (Gomes et al. 2002). O PEFI apresenta um mosaico de estádios sucessionais com manchas de áreas de estádio inicial de sucessão adjacentes a áreas de floresta secundária. Espécies arbóreas pioneiras como, Alchornea sidifolia Muell. Arg. e Cecropia glazioui Snethl, são abundantes nas bordas da floresta.

$\mathrm{O}$ estudo foi conduzido em duas áreas não contíguas dentro do PEFI. O principal critério de seleção foi a presença de Aulonemia aristulata (Döll) Macclure e o grau de cobertura deste bambu. $\mathrm{O}$ Parque de Ciências e Tecnologia (Cientec) (Área I), denominada floresta de dossel descontínuo/degradada por Peccinini \& Pivello (2002), onde o sub-bosque apresenta nível elevado de dominância por A. aristulata ( $>60 \%$ do sub-bosque) e o Instituto de Botânica (IBt) (Área II), denominada floresta de dossel heterogêneo e porte alto, onde $A$. aristulata ocorre de maneira mais esparsa como componente natural do sub-bosque. As áreas encontram-se detalhadamente descritas em Vinha et al. (2011).

Coleta de dados - Em cada área, foi estabelecido um bloco de 0,7 ha $(140 \times 50 \mathrm{~m})$, subdividido em 70 parcelas de $10 \times 10 \mathrm{~m}$. Todos os indivíduos de porte arbóreo (árvores, palmeiras e pteridófitas) com DAP $\geq 4,8 \mathrm{~cm}$, enraizados dentro de cada parcela, foram medidos, identificados e mapeados seguindo protocolo sobre implantação e medição de parcelas permanentes em área de Mata Atlântica (Joly et al. 2008).

Durante o período de novembro de 2008 a agosto de 2009 o censo de lianas foi realizado seguindo o protocolo estabelecido por Gerwing et al. (2006) e modificado por Schnitzer et al. (2008). Foram numerados, medidos, identificados e mapeados todos os sarmentos de lianas com diâmetro à altura do peito $(D A P) \geq 1,0 \mathrm{~cm}$ enraizados em cada parcela, que estivessem apoiados nos indivíduos arborescentes presentes na área. Para o cálculo de biomassa foi utilizada a equação alométrica desenvolvida por Schnitzer et al. (2006). Esta equação considera o diâmetro do sarmento à altura do peito $(130 \mathrm{~cm}$ do último ponto de enraizamento), com amostragem feita em parcelas:

$$
\ln (\mathrm{LAGB})=-1.484+2.657 * \ln (\mathrm{DAP}) \text {. }
$$

Sendo: LAGB a biomassa viva de lianas acima do solo e DAP: diâmetro medido à altura do peito.

A biomassa viva acima do solo dos indivíduos arbóreos nas áreas foi estimada segundo o modelo alométrico pantropical desenvolvido por Chave et al. (2005) para florestas tropicais úmidas e indicado por Vieira et al. (2008) como um modelo não-destrutivo adequado para estimar a biomassa de áreas de Mata Atlântica. Além do diâmetro (DAP), a equação pantropical incluiu variáveis como a altura $(\mathrm{H})$ e a densidade da madeira ou gravidade específica $(\rho)$.

$$
\ln (\mathrm{AAGB})=-2.977+\ln \left(\rho^{*} \mathrm{DAP}^{2 *} \mathrm{H}\right)
$$

A obtenção de estimativas sobre a porcentagem total de abertura do dossel (luminosidade média que atinge o solo) foi realizada através do registro de três fotos hemisféricas a $1,30 \mathrm{~m}$ de altura, no centro de cada sub-parcela de $10 \times 10 \mathrm{~m}$, com auxílio de máquina fotográfica digital e lentes hemisféricas Nikkon $8 \mathrm{~mm}$ (olho de peixe) por Carvalho et al. (dados não publicados). Para cada sub-parcela foi selecionada apenas a foto mais nítida dentre as três registradas. As fotos digitalizadas foram analisadas através do programa GLA (Gap Light Analyzer Frazer et al. 1999).

Análise dos dados - Para avaliar as diferenças na biomassa de lianas entre as áreas estudadas (em Mg. ha ${ }^{-1}$ ) realizou-se o teste de Mann-Whitney (Zar 1999). A comparação entre a proporção de indivíduos infestados entre as duas áreas foi realizada através do teste G (Zar 1999).

Lianas e árvores foram divididas em três classes de tamanho: pequeno diâmetro $(1,0 \leq \mathrm{A}<2,5 \mathrm{~cm})$, diâmetro intermediário $(2,5 \mathrm{~cm} \leq \mathrm{B}<5,0 \mathrm{~cm})$ e de grande diâmetro ( $\mathrm{C} \geq 5,0 \mathrm{~cm}$ ) (van Melis 2008). Para analisar a existência de autocorrelação espacial entre abundância e biomassa de lianas nas três classes de 
tamanho calculou-se o Índice de Moran (Legendre \& Legendre 1998) para cada parcela de $100 \mathrm{~m}^{2}$. Como a maioria das variáveis estruturais da floresta foi espacialmente correlacionada a curtas distâncias, aplicou-se então um teste parcial de Mantel para estimar as correlações, controlando o efeito da distância. As correlações foram realizadas a partir das matrizes de distância euclidiana, construídas com os valores de biomassa de árvores (AAGB) e de lianas (LAGB), abundância total de árvores e de lianas, abundância de lianas dentro de cada classe de tamanho; grau de abertura do dossel e altura do dossel. As correlações foram testadas através do teste parcial de Mantel utilizando-se o software Passage 1.1 (Rosenberg 2001) com 999 permutações e significância de 5\%.

\section{Resultados}

Um total de 1.031 lianas com diâmetro $\geq 1,0 \mathrm{~cm}$ foi amostrada nas duas áreas estudadas. No Cientec (Área I) foram amostrados 277 indivíduos em 0,7 ha de floresta (395 ind. ha $\left.{ }^{-1}\right)$. O diâmetro das lianas nesta área variou entre 1,0 e 13,9 cm. As lianas de grande diâmetro (classe $\mathrm{C}, \mathrm{DAP} \geq 5,0 \mathrm{~cm}$ ) contribuíram com $75 \%$ da LAGB, as com diâmetro intermediário (classe $\mathrm{B}, 2,5 \mathrm{~cm} \leq \mathrm{DAP}<5,0 \mathrm{~cm}$ ) totalizaram $24,2 \%$, e as de pequeno diâmetro (classe $\mathrm{A}, 1,0 \mathrm{~cm} \leq \mathrm{DAP}$ $<2,5 \mathrm{~cm}$ ) proporcionaram $4,52 \%$ da LAGB. Lianas da classe A de diâmetro foram as mais numerosas (52,3\% ou 145 dos 277 indivíduos amostrados) e lianas da classe de maior diâmetro foram as menos abundantes (15,5\% ou 43 dos 277) (figura 1).

No IBt, (Área II) foram amostrados 754 indivíduos em 0,7 ha de floresta (1.077 ind. ha $\left.{ }^{-1}\right)$. O diâmetro das lianas amostradas nesta área variou entre 1,0 e $16 \mathrm{~cm}$. As lianas de grande diâmetro (classe C, DAP $\geq 5,0 \mathrm{~cm}$ ) representaram $21,4 \%$ da LAGB, as de diâmetro intermediário (classe B, 2,5 $\leq$ DAP $<5,0 \mathrm{~cm}$ ) colaboraram com $30,9 \%$, e as lianas de pequeno diâmetro (classe $\mathrm{A}, 1,0 \leq \mathrm{DAP}<2,5 \mathrm{~cm}$ ) contribuíram com 47,6\% da LAGB . As lianas da classe A foram mais abundantes $(50,6 \%$ dos 754 indivíduos amostrados) do que lianas da classe $\mathrm{C}$, com apenas 10,74\% ou 81 dos 754 indivíduos (figura 1). A proporção de indivíduos dentro da classe de maior diâmetro $(\geq 5,0 \mathrm{~cm})$ é significativamente diferente entre as áreas $(\mathrm{U}=4,15, \mathrm{p}=0,04, \mathrm{gl}=1)$.

Houve diferenças significativas $(\mathrm{U}=62,8282$, $\mathrm{p}<0.0001, \mathrm{gl}=1$ ) entre as áreas quanto à proporção
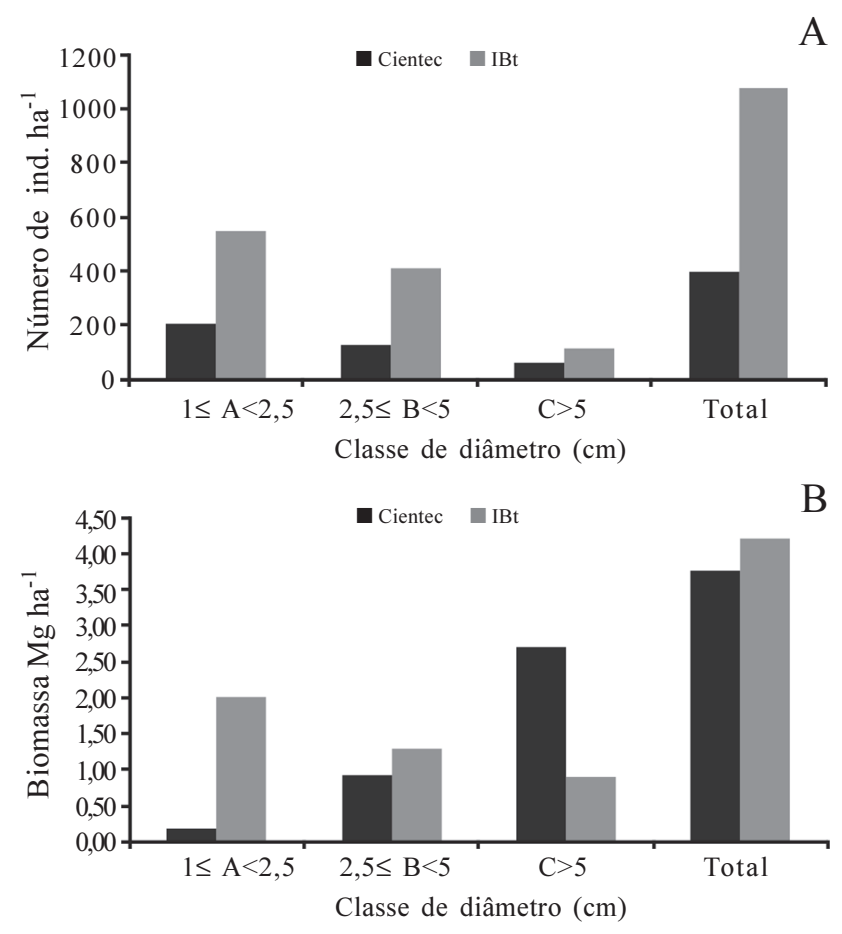

Figura 1. Distribuição da densidade (ind ha ${ }^{-1}$ ) (A) e distribuição de biomassa $\left(\mathrm{Mg} \mathrm{ha}^{-1}\right)(\mathrm{B})$ em classes de diâmetro nas áreas do Parque de Ciências e Tecnologia (Cientec): dominada por bambu e Instituto de Botânica (IBt) localizadas no Parque Estadual das Fontes do Ipiranga (PEFI), São Paulo.

Figure 1. Density distribution of (ind $\mathrm{ha}^{-1}$ ) (A) and biomass distribution $\left(\mathrm{Mg} \mathrm{ha}^{-1}\right)$ across diameter classes for the Parque de Ciências e Tecnologia (Cientec): bamboo dominated and Instituto de Botânica (IBt) located at the Parque Estadual das Fontes do Ipiranga (PEFI), São Paulo.

de indivíduos infestados: 15\% na área I (Cientec) e $30 \%$ na área II (IBt) e quanto à biomassa total de lianas $(U=5,1511, p<0,0001)$. Os valores de biomassa de lianas encontrado nas duas áreas amostradas foram de 3,76 e 4,2 $\mathrm{Mg} \mathrm{ha}^{-1}$ respectivamente para as áreas I e II.

Embora as duas áreas amostradas apresentem diferenças estruturais na densidade (1.354 caules ha ${ }^{-1}$ e 1.767 caules $\mathrm{ha}^{-1}$ - áreas I e II respectivamente) e na biomassa de árvores $\left(186,45 \mathrm{Mg} \mathrm{ha}^{-1}\right.$ e 203,06 $\mathrm{Mg} \mathrm{ha}^{-1}$ - áreas I e II respectivamente) e na densidade de palmeiras (50 caules ha $\mathrm{ha}^{-1}$ e 24,3 caules ha-1 respectivamente para áreas I e II) (teste de MannWhitney, $\mathrm{p}<0,05$ para todas as comparações) não foram encontradas correlações entre a estrutura da floresta e características estruturais das lianas (abundância e biomassa). $\mathrm{Na}$ área II, foram encontradas baixas correlações entre a biomassa de lianas e a biomassa de árvores $\left(r_{\text {Parcial }}=0,24\right)$, entre a abundância de lianas e a 
abundância total de árvores $\left(r_{\text {Parcial }}=0,02\right)$, e entre a abundância de lianas e o grau de abertura do dossel $\left(r_{\text {Parcial }}=-0,02\right)$, que foi significativamente menor na área II $(8,10 \pm 0,36)$ que na área I $(12,05 \pm 0,42)$ segundo os resultados dos testes parciais de Mantel. A biomassa aérea de lianas (LAGB) apresentou correlação significativa, segundo os testes parciais de Mantel, com a abundância total de lianas e com a biomassa das classes de diâmetro médio e grande (B e C), tanto para $\mathrm{a}$ área I quanto para a área II (tabela 1).

Tabela 1. Valores de correlação com o teste parcial de Mantel controlando o efeito da distância. LAGB biomassa aérea de lianas; Lianas médias: 2,5-4,9 cm; Lianas grandes ( $\geq 5,0 \mathrm{~cm})$.

Table 1. Correlation values with the partial Mantel test controlling the distance effect. LAGB aboveground biomass of lianas; Medium size lianas: 2.5 to $4.9 \mathrm{~cm}$; Large Lianas $(\geq 5.0 \mathrm{~cm})$.

\begin{tabular}{lcll}
\hline Área & Matriz 1 & Matriz 2 & $r_{\text {Parcial }}$ \\
\hline IBt & Lianas médias & LAGB & $0,21^{*}$ \\
& Lianas grandes & LAGB & $0,47^{* *}$ \\
Cientec & Lianas médias & LAGB & $0,14^{*}$ \\
& Lianas grandes & LAGB & $0,62^{* *}$ \\
\hline
\end{tabular}

Probabilidades dos $r$-valores de Mantel $\left({ }^{*} \mathrm{p}<0,01\right.$; **p $\left.<0,001\right)$ foram obtidos após 999 permutações.

\section{Discussão}

A maior abundância de lianas de menor porte é um padrão comum encontrado em outras florestas. Lianas com diâmetro entre $1,0 \mathrm{~cm}$ e $2,5 \mathrm{~cm}$ representaram $69,3 \%$ dos indivíduos amostrados na Reserva Ducke, na Amazônia (Nogueira et al. 2010) e $63,87 \%$ dos indivíduos amostrados em Ubatuba (van Melis 2008). De maneira geral, lianas de diâmetro grande são mais comuns em áreas menos perturbadas e de estádio sucessional mais avançado (Laurance et al. 2001, Rice et al. 2004, Letcher \& Chazdon
2009) pois dependem de grandes árvores para sustentá-las e proporcionarem acesso a luz (Philips et al. 2005). Fatores ambientais como, fertilidade do solo e a estrutura da floresta podem atuar como filtros ecológicos, selecionando indivíduos durante o desenvolvimento e favorecendo a abundância de lianas de classes de tamanho pequeno que são as mais abundantes (Nogueira et al. 2010). Além disso, a área basal das lianas pode estar relacionada à composição distinta de espécies entre as áreas amostradas (Garrido-Pérez et al. 2008). Entretanto, embora em ambas as áreas haja uma maior abundância de indivíduos da menor classe de diâmetro, uma proporção significativamente maior de lianas de grande porte foi encontrada na área I (Cientec), dominada por bambus. Este resultado pode ser explicado pela existência nessa área, de cerca de $6 \%$ de árvores com diâmetro superior a $30 \mathrm{~cm}$ que podem sustentar lianas de diâmetro maior. Indivíduos entre 10 e $30 \mathrm{~cm}$ de diâmetro concentraram uma maior proporção dos indivíduos (44\%) na área I.

Os valores de biomassa de lianas encontrados em ambas as áreas estão abaixo dos valores estimados em outros estudos (tabela 2). Entretanto, em cada um desses trabalhos, os autores utilizaram diferentes equações para estimar a biomassa o que restringe as comparações.

As diferenças estruturais encontradas entre as duas comunidade de lianas - maior taxa de infestação de caules por lianas, maior abundância e biomassa de lianas e menor proporção de lianas de diâmetro grande na área II, não-dominada por bambus - corroboram as diferenças estruturais na biomassa e densidade de árvores, além da densidade de palmeiras entre as áreas. Sabe-se que, dentre os fatores relacionados à dominância de lianas, destacam-se tanto a ocorrência de pequenos quanto de grandes distúrbios, sejam eles de origem natural ou antrópica (Schnitzer \& Bongers 2002). De maneira geral, tem-se que a abundância de

Tabela 2. Estimativa de biomassa de lianas em áreas de Floresta Tropical. Lianas: critério de amostragem; *Somente trabalhos que informaram não incluir hemi-epífitas.

Table 2. Biomass estimation of lianas in tropical forest areas. Lianas: sampling criterion;* Only papers that did not include hemiepyphites were reported in this table.

\begin{tabular}{|c|c|c|c|}
\hline Estudo & Localidade & Lianas & Biomassa \\
\hline Putz (1983) & Venezuela & $\begin{array}{c}\text { lianas } \geq 2,0 \mathrm{~m} \\
\text { altura }\end{array}$ & $15,7 \mathrm{Mg} \mathrm{ha}^{-1}$ \\
\hline Gerwing \& Farias (2000) & Floresta Amazônica & $\begin{array}{c}\text { lianas } \geq 2,0 \mathrm{~m} \\
\text { altura }\end{array}$ & $43 \mathrm{Mg} \mathrm{ha}^{-1}$ \\
\hline Nascimento \& Laurance (2002) & Floresta Amazônica & lianas $\geq 2,0 \mathrm{~cm}$ & $4,6-13,7 \mathrm{Mg} \mathrm{ha}^{-1}$ \\
\hline DeWalt \& Chave (2004) & Panamá, Costa Rica e Peru & lianas $\geq 0,5 \mathrm{~cm}$ & $8,0-17,2 \mathrm{Mg} \mathrm{ha}^{-1}$ \\
\hline
\end{tabular}


lianas está diretamente relacionada ao aumento na abundância de árvores, visto que as mesmas servem como suporte para este grupo de plantas (Nabe-Nielsen 2001, Philips et al. 2002, Addo-Fodjour et al. 2009). Entretanto, a abundância de lianas é negativamente correlacionada com a abundância de palmeiras, uma vez que estas não são caracterizadas como um suporte para a escalada de lianas (Nogueira et al. 2010).

Para a área I, além de uma menor disponibilidade de árvores como suporte, outro fator que pode ter contribuído para a diminuição na biomassa e abundância de lianas em relação á área II foi a competição por luz e espaço com Aulonemia aristulata, uma espécie de bambu nativo escandente que forma uma extensa cobertura nas árvores e que durante 17 anos dominou o local (Vinha et al. 2011). O sub-bosque da área I (ca. de 1 ha) é dominado por Aulonemia aristulata (Döll) McClure Poaceae: Bambusoideae), uma espécie de bambu lenhoso que ocorre em florestas úmidas e sazonais nas região central e no sudeste do Brasil. De maneira geral essa espécie apresenta populações descontínuas e em baixa densidade; entretanto, pode alcançar altas densidades em bordas e áreas de dossel aberto e dominar áreas mais perturbadas (Vinha et al. 2011). Segundo Clark (2001), A. aristulata é caracterizada por apresentar rizoma anfimorfo (ver Judziewicz et al. 1999), colmos decumbentes ou escandentes com 1-4 m, entrenós ocos, e nós no meio do colmo com uma gema, resultando no complemento de um só ramo dominante, portando na base 2 a muitos ramos bem desenvolvidos e livres. Dessa forma, lianas podem ter seu crescimento suprimido pela competição por bambus (Campanello et al. 2007) na área I, dominada pelo bambu.

Nossa hipótese inicial de que a área dominada por bambus apresentaria uma menor densidade e biomassa de lianas em função da baixa disponibilidade de árvores para suporte e da competição com o bambu nativo Aulonemia aristulata foi confirmada. Os resultados obtidos por este estudo ratificam as informações de que perturbações na floresta e aumento no número de clareiras não conduzem necessariamente a um aumento na abundância de lianas (AddoFodjour et al. 2008, 2009) sendo esta maior nos estádios intermediários de sucessão (Ladwig \& Meiners 2010) devido a um balanço tanto entre a disponibilidade de suportes quanto a de luz (Madeira et al. 2009). Entretanto, é preciso considerar que áreas onde bambus aparecem como espécies dominantes apresentam alterações estruturais que devem fortemente influenciar a dinâmica florestal.

\section{Agradecimentos}

Este trabalho foi financiado pela Fundação de Amparo à Pesquisa do Estado de São Paulo, Proc. n. 05/51747-2. Paula Guimarães Carvalho foi bolsista de IC do Programa Institucional PIBIC /CNPq, Fernando Mancini Cestari foi bolsista IC da Fapesp (Proc. n. 08/57784-5) e Bruno Marques Ascenção foi bolsista de TT-3 da Fapesp (Proc. n. 08/02939-4).

\section{Literatura citada}

Addo-Fordjour, P., Anning, A.K., Atakora, E.A. \& Agyei, P.S. 2008. Diversity and distribution of climbing plants in a semi-deciduous rain forest, KNUST Botanic Garden, Ghana. International Journal of Botany 4: 186-195.

Addo-Fordjour, P., Anning, A.K., Larbi, J.A. \& Akyeampong, S. 2009. Liana species richness, abundance and relationship with trees in the Bobiri forest reserve, Ghana: impact of management systems. Forest Ecology and Management 257: 1822-1828.

Barros, F., Mamede, M.C.H., Melo, M.M.R.F., Lopes, E., Jung-Mendaçolli, S., Kirizawa, M., Muniz, C.F.S., Makino-Watanabe, H., Chiea, S.A.C. \& Melhem, T.S. 2002. A Flora Fanerogâmica do PEFI: composição, afinidades e conservação. In: D.C. Bicudo, M.C. Forti \& C.E.M. Bicudo (eds.). Parque Estadual das Fontes do Ipiranga (PEFI): unidade de conservação que resiste à urbanização de São Paulo. Secretaria do Meio Ambiente, São Paulo, pp. 93-110.

Campanello, P.I., Gatti, M.G.,Ares,A., Montti,L. \& Goldstein, G. 2007. Tree regeneration and microclimate in a liana and bamboo-dominated semideciduous Atlantic Forest. Forest Ecology and Management 252: 108-117.

Chave, J., Andalo, C., Brown, S., Cairns, M.A., Chambers, J.Q., Eamus, D., Fölster, H., Fromard, F., Higuchi, N., Kira, T., Lescure, J.P., Nelson, B.W., Ogawa, H., Puig, H., Riéra, B. \& Yamakura, T. 2005. Tree allometry and improved estimation of carbon stocks and balance in tropical forests. Oecologia 145: 87-99.

Clark, L.G. 2001. Aulonemia. In: M.G.L. Wanderley, G.J. Shepherd, A.M. Giulietti \& T.S. Melhem (eds.). Flora Fanerogâmica do Estado de São Paulo. Hucitec, São Paulo, v.1, pp. 127-145.

Dewalt, S.J., \& Chave, J.C. 2004. Structure and biomass of four lowland Neotropical forests. Biotropica 36: 7-19.

DeWalt, S.J., Schnitzer , S.A., Chave, J., Bongers, F., Burnham, Robyn, J., Cai, Z., Chuyong, G., Clark, D.B., Ewango, C.E.N., Gerwing, J.J., Gortaire, E., Hart, T., Ibarra-Manríquez, G., Ickes, K., Kenfack, D., Macía, M.J., Makana, J.R., Martínez-Ramos, M., Mascaro, J., Moses, S., Muller-Landau, H.C., Parren, M.P.E., Parthasarathy, N., Pérez-Salicrup, D.R., Putz, F.E., Romero-Saltos, H. \& Thomas, D. 2010. Annual rainfall and seasonality predict Pan-tropical patterns of liana density and basal area. Biotropica 42: 309-327. 
Embrapa. 1999. Solos. Sistema brasileiro de classificação de solos. Empresa Brasileira de Pesquisa Agropecuária, Brasília.

Engel, V.L., Fonseca, R.C.B. \& Oliveira, R.E. 1998. Ecologia de lianas e o manejo de fragmentos florestais. Série Técnica IPEF 12: 43-64.

Frazer, G.W., Canham, C.D. \& Lertzman, K.P. 1999. Gap Light Analyzer (GLA) Version 2.0: Imaging software to extract canopy structure and gap light transmission indices from true-colour fisheye photographs: users manual and program documentation. Simon Fraser University, Burnaby Institute of Ecosystems Studies, Millbrook.

Garrido-Pérez, E.I., Dupuy, J.M., Drán-Garcia, R., UcanMay, M., Schitzer, S.A. \& Gerold, G. 2008. Effects of lianas and hurricane Wilma on tree damage in the Yucatan peninsula, Mexico. Journal of Tropical Ecology 24: 559-562.

Gentry, A.H. 1983. Lianas and the "paradox" of contrasting latitudinal gradients in wood and litter production. Tropical Ecology 24: 63-67.

Gentry, A.H. \& Dodson, C. 1987. Contribution of nontrees to species richness of a tropical rain forest. Biotropica 19: 149-156.

Gerwing, J.J. \& Farias, D.L. 2000. Integrating liana abundance and forest stature into an estimate of aboveground biomass for an eastern Amazonian forest. Journal of Tropical Ecology 16: 327-336.

Gerwing, J.J., Schnitzer, S.A., Burnham, R.J., Bongers, F., Chave, J., Dewalt, S.J., Ewango, C.E.N., Foster, R., Kenfack, D., Martinez-Ramos, M., Parren, M., Parthasarathy, N., Perez-Salicrup, D.R., Putz, F.E. \& Thomas, D.W. 2006. A standard protocol for lianas censuses. Biotropica 38: 256-261.

Gomes, E.P.C., Kageyama, P.Y. \& Mantovani, W. 2002. Dinâmica da floresta no PEFI. In: D.C. Bicudo, M.C. Forti \& C.E.M. Bicudo (eds.). Parque Estadual das Fontes do Ipiranga (PEFI): unidade de conservação que resiste à urbanização de São Paulo. Secretaria do Meio Ambiente, São Paulo, pp. 111-132.

González, M.E., Veblen, T.T., Donoso, C. \& Valeria, L. 2002. Tree regeneration responses in a lowland Notophagusdominated forest after bamboo dieback in South-Central Chile. Plant Ecology 161: 59-73.

Granados, J. \& Körner, C. 2002. In deep shade, elevated $\mathrm{CO}_{2}$ increases the vigor of tropical climbing plants. Global Change Biology 8: 1109-1117.

Griscom, B.W. \& Ashton, P.M.S. 2003. Bamboo control of forest succession: Guadua sarcocarpa in Southeastern Peru. Forest Ecology and Management 175: 445-454.

Ingwell, L.L., Wright, S.J., Becklund, K.K., Hudbell, S.P. \& Schnitzer, S.A. 2010. The impact of lianas on 10 years of tree growth and mortality on Barro Colorado Island, Panama. Journal of Ecology 98: 879-887.
Joly, C.A., Martinelli, L.A., Alves, L.F., Vieira, S.A., Tamashiro, J.Y., Aidar, M.P.M., Camargo, P.B., Assis, M.A. \& Bernacci, L.C. 2008. As parcelas permanentes do Projeto Temático BIOTA Gradiente Funcional: Composição florística, estrutura e funcionamento da Floresta Ombrófila Densa dos Núcleos Picinguaba e Santa Virgínia do Parque Estadual da Serra do Mar, São Paulo, Brasil. In: C.O. Sanquetta (org.). Experiências de monitoramento no bioma Mata Atlântica com uso de parcelas permanentes. Rede de Parcelas Permanentes dos Biomas Mata Atlântica e Pampa \& Fundação Universidade do Paraná, Curitiba, pp. 109-148.

Judziewicz, E.J., Clarck, L.G., Londoño, X. \& Stern, M.J. 1999. American bamboos. Smithsonian Institute Press, Washington.

Kapos, V., Wandelli, E., Camargo, J.L. \& Ganade, G. 1997. Edge-related changes in environment and plant responses due to forest fragmentation in central Amazonia. In: W.F. Laurance \& R. Bierregaard Jr. (eds.). Tropical forest remnants: ecology management, and conservation of fragmented communities. The University of Chicago Press, Chicago, pp. 33-45.

Köppen, W. 1948. Climatologia. Fondo de Cultura Económica. México.

Ladwig, L.M. \& Meiners, S.J. 2010. Liana host preference and implications for deciduous forest regeneration. Journal of the Torrey Botanical Society 137: 103-112.

Laurance, W.F. 1997. Hyper-disturbed parks: edge effects and the ecology of isolated rainforest reserves in tropical Australia. In: W.F. Laurance \& R.O. Bierregaard Jr. (eds.). Tropical forest remnants: ecology management, and conservation of fragmented communities. The University of Chicago Press, Chicago.

Laurance, W.F., Pérez-Salicrup, D., Delamônica, P., Fearnside, P., D'Angelo, S., Jerozolinski, A., Pohl, L. \& Lovejoy, T.E. 2001. Rain forest fragmentation and the structure of Amazonian liana communities. Ecology 82: 105-116.

Legendre, P. \& Legendre, L. 1998. Numerical Ecology. Elsevier, Amsterdam.

Letcher, S.G. \& Chazdon, R.L. 2009. Lianas and selfsupporting plants during forest succession. Forest Ecology and Management 257: 2150-2156.

Madeira, B.G., Santo, M.M.E., Neto, S.A., Nunes, Y.R.F., Azofeifa, G.A.S., Fernandes, G.W. \& Quesada, M. 2009. Changes in tree and liana communities along a successional gradient in a tropical dry forest in southeastern Brazil. Plant Ecololgy 201: 291-304.

Malizia, A. \& Grau, H.R. 2006. Liana-host tree associations in a subtropical montane forest of north-western Argentina. Journal of Tropical Ecology 22: 331-339.

Nabe-Nielsen, J. 2001. Diversity and distribution of lianas in a neotropical rain forest, Yasuní National Park, Ecuador. Journal of Tropical Ecology 17: 1-19.

Nascimento, H.E.M. \& Laurance, W.F. 2002. Total aboveground biomass in central Amazonian rainforests: a landscape-scale study. Forest Ecology and Management 168: 311-321. 
Nastri, V.D.F., Catharino, E.L.M., Rossi, L., Barbosa, L.M., Pirré, E., Bedinelli, C., Asperti, L.M., Dorta, R.O. \& Costa, M.P. 1992. Estudos fitossociológicos de uma área do Instituto de Botânica de São Paulo utilizada em programa de educação ambiental. In: J.L. Timoni (ed.). Anais do II Congresso Nacional sobre Essências Nativas. Revista do Instituto Florestal. 4:219-225.

Nogueira, A., Costa, F.R.C. \& Castilho, C.V. 2010. Liana Abundance Patterns: The Role of Ecological Filters during Development. Biotropica. Disponível em http:// onlinelibrary.wiley.com/doi/10.1111/j.17447429.2010.00722.x/full (acesso em 1.06.2010).

Oliveira Filho, A.T. \& Fontes, M.A.L. 2000. Patterns of floristic differentiation among Atlantic Forest in Southeastern Brazil and the influence of climate. Biotropica 32: 793-810.

Oosterhoorn, M. \& Kappelle, M. 2000. Vegetation structure and composition along an interior-edge-exterior gradient in a Costa Rican montane cloud forest. Forest Ecology and Management 126: 291-307.

Phillips, O.L., Martínez, R.V., Arroyo, L., Baker, T.R., Killeen, T., Lewis, S.L., Malhi, Y., Mendoza, A.M., Neill, D., Vargas, P.N., Alexiades, M., Cerón, C., Di Fiore, A., Erwin, T., Jardim, A., Palacios, W., Saldias, M. \& Vinceti, B. 2002. Increasing dominance of large lianas in Amazonian forests. Nature 418: 770-774.

Phillips, O.L., Martinez, R.V., Mendoza,A.M., Baker, T.R. \& Vargas, P.N. 2005. Large lianas as hyperdynamic elements of the tropical forest canopy. Ecology 85: 1250-1258.

Pivelo, V.R. \& Peccinini, A.A. 2002. A Vegetação do PEFI. In: D.C. Bicudo, M.C. Forti \& C.E.M. Bicudo (eds.). Parque Estadual das Fontes do Ipiranga (PEFI): unidade de conservação que resiste à urbanização de São Paulo. Secretaria do Meio Ambiente, São Paulo, pp. 75-92.

Putz, F.E. 1983. Liana biomass and leaf area of a tierra firme forest in the Rio Negro basin, Venezuela. Biotropica 15: 185-89.

Putz, F.E. 1984. The natural history of lianas on Barro Colorado Island, Panama. Ecology 65: 1713-1724.

Rice, K., Brokaw, N. \& Thompson, J. 2004. Liana abundance in a Puerto Rican Forest. Forest Ecology and Management 190: 33-41.

Rosenberg, M.S. 2001. PASSAGE. Pattern Analysis, Spatial Statistics, and Geographic Exegesis. Version 1.1 (Release 3.4). Department of Biology, Arizona State University. Disponível em http://www.passagesoftware.net (acesso em 18.11.2009).

Sarmiento, G., Pinillos, M. \& Garay, I. 2005. Biomass variability in tropical American lowland Rainforest. Ecotropicos 18: 1-20.

Schnitzer, S.A. \& Bongers, F. 2002. The ecology of lianas and their role in forests. Trends in Ecology and Evolution 17:223-230.

Schnitzer, S.A., Dalling, J.W. \& Carson, W.P. 2000. The impact of lianas on tree regeneration in tropical forest canopy gaps: evidence for an alternative pathway of gap-phase regeneration. Journal of Ecology 88: 655-666.
Schinitzer, S.A., Dewalt, S.J. \& Chave, J. 2006. Censusing and measuring lianas: a quantitative comparison of the common methods. Biotropica 38: 581-591.

Schnitzer, S.A., Rutishauser, S. \& Salomón, A. 2008. Supplemental protocol for liana censuses. Forest Ecology and Management 255: 1044-1049.

Senbeta, F., Schmitt, C., Denich, M., Demissew, S., Vlek, P.I.G., Preisinger, H., Woldemariam, T. \& Teketay, D. 2005. The diversity and distribution of lianas in the Afromontane rain forests of Ethiopia. Diversity and Distribution 11: 443-452.

Tabarelli, M. \& Mantovani, W. 1999. A regeneração de uma floresta tropical montana após corte e queima (São PauloBrasil). Revista Brasileira de Botânica 22: 217-223.

Van der Heijden, G.M.F., Healey, J.R. \& Phillips, O.L. 2008. Infestation of trees by lianas in a tropical forest in Amazonian Peru. Journal of Vegetation Science 19: 747-756.

Van Melis, J. 2008. Lianas: biomassa em florestas neotropicais e relação a riqueza e biomassa em um trecho de Floresta Ombrófila Densa Atlântica. Dissertação de Mestrado, Universidade estadual de Campinas, Campinas.

Veloso, H.P., Rangel Filho, A.L.R. \& Lima, J.C.A. 1991. Classificação da vegetação brasileira adaptada a um sistema universal. IBGE, Rio de Janeiro.

Victor, A.B.M., Costa Neto, J.B., Ab'Saber, A.N., Serrano, O., Domingos, M., Pires, B.C.C., Amazonas, M. \& Victor, M.A.M. 2004. Application of the biosphere reserve concept to urban areas. The case of São Paulo city green belt biosphere reserve, Brazil - São Paulo forest institute: a case study for UNESCO. Annals of New York Academy of Sciences 1023: 237-281.

Vieira, S.A., Alves, L.F., Aidar, M.P.M., Araújo, L.S., Baker, T., Batista, J.L.F., Campos, M.C.R., Camargo, P.B., Chave, J., Delitti, W.B., Higuchi, N., Honório, E., Joly, C.A., Keller, M., Martinelli, L.A., Mattos, E.A., Metzker, T., Phillips, O.L., Santos, F.A.M., Shimabukuro, M.T., Silveira, M. \& Trumbore, S.E. 2008. Estimation of biomass and carbon stocks: the case of the Atlantic Forest. Biota Neotropica 8. Disponível em http:// w w w.biotan e tropica.org.br/v $8 \mathrm{n} 2 / \mathrm{pt} /$ fullpaper?bn00108022008+en.doi: 10.1590/S167606032008000200001 (acesso em 14.03.2011).

Vinha, D., Alves, L.F., Zaidan, L.B.P. \& GromboneGuaratini, M.T. 2011. The soil seed bank after 7 years of bamboo dominance in a Tropical Forest in SE Brazil. Landscape and Urban Planning 99: 178-185.

Wang, W., Franklin, S.B., Ren, Y. \& Ouellette, J.R. 2006. Growth of bamboo Fargesia qinlingensis and regeneration of trees in a mixed hardwood-conifer forest in the Qinling Mountains, China. Forest Ecology and Management 234: 107-115.

Wright, S.J., Calderón, O., Hernandéz, A. \& Paton, S. 2004. Are lianas increasing in importance in tropical forest? Ecology 85: 484-489.

Zar, J.H. 1999. Biostatistical analysis. Prentice Hall, Upper Saddle River.

Zhu, S.D. \& Cao, K.F. 2010. Contrasting cost-benefit strategy between lianas and trees in a tropical seasonal rain forest in southwestern China. Oecologia 163: 591-599. 